\title{
Nuclear Dynamics at the Balance Energy
}

\author{
Aman D. Sood and Rajeev K. Puri \\ Physics Department, Panjab University, Chandigarh-160 014, India
}

December 5, 2018

\begin{abstract}
We study the mass dependence of various quantities (like the average and maximum density, collision rate, participant-spectator matter, temperature as well as time zones for higher density) by simulating the reactions at the energy of vanishing flow. This study is carried out within the framework of Quantum Molecular Dynamics model. Our findings clearly indicate an existence of a power law in all the above quantities calculated at the balance energy. The only significant mass dependence was obtained for the temperature reached in the central sphere. All other quantities are rather either insensitive or depend weakly on the system size at balance energy. The time zone for higher density as well as the time of maximal density and collision rate follow a power law inverse to the energy of vanishing flow.
\end{abstract}

\section{Introduction}

It is now well established that the interactions at low incident energies are dominated by the attractive part of the nuclear mean field causing the emission of particles in the backward 
angles. These interactions, however, become repulsive at higher incident energies that pushes the particles in the forward (positive) angles. While going from the low incident energies to higher incident energies, there is a particular energy at which the net flow disappears [1]. This energy, (termed as "balance energy") $E_{b a l}$ has been found to be of significant importance for the understanding of the nature of nuclear interactions and related dynamics [2-24].

Recently, the $E_{b a l}$ was measured in ${ }^{197} \mathrm{Au}+{ }^{197} \mathrm{Au}$ collisions [4, 5], extending the mass range of $E_{b a l}$ between 24 and 394 units. In addition to ${ }^{197} \mathrm{Au}+{ }^{197} \mathrm{Au}$ collisions, one has also measured the $E_{b a l}$ in ${ }^{12} \mathrm{C}+{ }^{12} \mathrm{C}$ [7], ${ }^{20} \mathrm{Ne}+{ }^{27} \mathrm{Al}$ [7], ${ }^{36} \mathrm{Ar}+{ }^{27} \mathrm{Al}$ [9, 12,,${ }^{40} \mathrm{Ar}+{ }^{27} \mathrm{Al}\left[8\right.$ ], ${ }^{40} \mathrm{Ar}+{ }^{45} \mathrm{Sc}$ [5, 7, 13], ${ }^{40} \mathrm{Ar}+{ }^{51} \mathrm{~V} \mathrm{[10]},{ }^{64} \mathrm{Zn}+{ }^{27} \mathrm{Al}[11],{ }^{40} \mathrm{Ar}+{ }^{58} \mathrm{Ni}$ [6], ${ }^{64} \mathrm{Zn}+{ }^{48} \mathrm{Ti}$ [12, ${ }^{58} \mathrm{Ni}+{ }^{58} \mathrm{Ni}$ [5, 6, 13], ${ }^{58} \mathrm{Fe}+{ }^{58} \mathrm{Fe}$ [13, ${ }^{64} \mathrm{Zn}+{ }^{58} \mathrm{Ni}\left[12,{ }^{86} \mathrm{Kr}+{ }^{93} \mathrm{Nb}\right.$ [5, 7], ${ }^{93} \mathrm{Nb}+{ }^{93} \mathrm{Nb}$ [3], ${ }^{129} \mathrm{Xe}+{ }^{118} \mathrm{Sn}$ [6] and ${ }^{139} \mathrm{La}+{ }^{139} \mathrm{La}$ ] collisions. Most of the above reactions are symmetric and central in nature. Some attempts are also reported in the literature that deal with the impact parameter dependence of the balance energy $E_{b a l}$ [5, 6, 8, 11, 13, 17, 20, 21].

Interestingly, most of the theoretical attempts for disappearance of flow are within the Boltzmann-Uehling-Uhlenbeck (BUU) model [1, 3, 4, 15, 7, 8, 11, 13, 15, 16, 17, 18. Some attempts, however, are also made within the framework of Quantum Molecular Dynamics (QMD) model [13, 19, 20, 21, 22, 23, 24]. Note that among all these attempts, only a few deal with the mass dependence of the disappearance of flow [4, 5, 17, 18, 22, 23, 24. There one reported a power law behavior $\left(\propto A^{\tau}\right)$ in the $E_{b a l}$. For the first time, a complete study of the mass dependence of balance energy was presented by us where as many as sixteen systems, with mass between 47 and 476 were analyzed [22, 23, 24]. The excellent agreement between the experimental measurements and theoretical calculations allowed us to predict the balance energy in ${ }^{238} \mathrm{U}+{ }^{238} \mathrm{U}$ collision around 37-39 MeV/nucleon [22]. None of the above studies was extended to other heavy-ion phenomena at balance energy. Refs. [11, 15, 18, 20, 21, give some 
information about the nature of other variables at the balance energy.

We here present a complete analysis of the nuclear dynamics at balance energy by analyzing more than 14 (nearly symmetric) reactions as reported in ref. 23, 24. Our present motivation is to investigate whether other dynamical variables (apart from the disappearance of flow) show a mass dependence at the balance energy or not. This present study is made within the framework of Quantum Molecular Dynamics (QMD) model [19, 20, 21, 22, 23, 24, 25, 26, 27, 28, 29]. Section II describes the model in brief. Our results along with the discussion are presented in section III. We summarize the results in section IV.

\section{The Model}

The present study is made within the framework of the Quantum Molecular Dynamics (QMD) model [21, 25, 26] where each nucleon interacts via two- and three-body interactions that preserve the nucleon-nucleon (nn) correlations and fluctuations. Here each nucleon is represented by a Gaussian wave packet with width $\sqrt{L}$ centered around the mean position $\vec{r}_{i}(t)$ and the mean momentum $\vec{p}_{i}(t)$ :

$$
\phi_{i}(\vec{r}, \vec{p}, t)=\frac{1}{(2 \pi L)^{3 / 4}} e^{\left[-\left(\vec{r}-\vec{r}_{i}(t)\right)^{2} / 4 L\right]} e^{\left[i \vec{p}_{i}(t) \cdot \vec{r} / \hbar\right]}
$$

The Wigner distribution of a system with $\left(A_{T}+A_{P}\right)$ nucleons is given by:

$$
\begin{aligned}
f(\vec{r}, \vec{p}, t)= & \sum_{i=1}^{A_{T}+A_{P}} \frac{1}{(\pi \hbar)^{3}} e^{\left[-\left(\vec{r}-\vec{r}_{i}(t)\right)^{2} / 2 L\right]} \times \\
& e^{\left[-\left(\vec{p}-\vec{p}_{i}(t)\right)^{2} 2 L / \hbar^{2}\right]} .
\end{aligned}
$$

The nucleons propagate under the classical equations of motion:

$$
\frac{d \vec{r}_{i}}{d t}=\frac{\partial\langle H\rangle}{\partial \vec{p}_{i}}
$$




$$
\frac{d \vec{p}_{i}}{d t}=-\frac{\partial\langle H\rangle}{\partial \vec{r}_{i}}
$$

The Hamiltonian $\langle H\rangle$ is given by:

$$
\begin{aligned}
\langle H\rangle & =\langle T\rangle+\langle V\rangle \\
& =\sum_{i} \frac{\vec{p}_{i}^{2}}{2 m_{i}}+\sum_{i} \sum_{i<j} V_{i j}^{\text {total }},
\end{aligned}
$$

with

$$
V_{i j}^{\text {total }}=V_{i j}^{\text {local }}+V_{i j}^{Y u k}+V_{i j}^{\text {Coul }}
$$

Here $V_{i j}^{\text {local }}, V_{i j}^{\text {Coul }}$ and $V_{i j}^{Y u k}$ stand, respectively, for the Skyrme, Coulomb and Yukawa parts of the nn interaction. Following refs. [22, 23, 24, we use a hard equation of state along with energy independent cross-section of $40 \mathrm{mb}$ strength. This combination is reported to explain the experimental balance energy nicely [23].

\section{Results and Discussion}

As stated in ref. [23], a hard equation of state along with energy independent nn cross-section of $40 \mathrm{mb}$ strength yields a power law behavior $\propto \mathrm{A}^{\tau}$. The power law $\left(c . A^{\tau}\right)$ over the experimental points yields $\tau=-0.42079 \pm 0.04594$, whereas our theoretical calculations with nn cross-section of $40 \mathrm{mb}$ strength had $\tau=-0.41540 \pm 0.08166$ [23]. It is worth mentioning that this was the closest agreement obtained so far. For the present mass dependent analysis, we simulated the reactions of ${ }^{20} \mathrm{Ne}+{ }^{27} \mathrm{Al}(\mathrm{b}=2.6103 \mathrm{fm}),{ }^{36} \mathrm{Ar}+{ }^{27} \mathrm{Al}(\mathrm{b}=2 \mathrm{fm}),{ }^{40} \mathrm{Ar}+{ }^{27} \mathrm{Al}(\mathrm{b}=1.6 \mathrm{fm})$, ${ }^{40} \mathrm{Ar}+{ }^{45} \mathrm{Sc}(\mathrm{b}=3.187 \mathrm{fm}),{ }^{40} \mathrm{Ar}+{ }^{51} \mathrm{~V}(\mathrm{~b}=2.442 \mathrm{fm}),{ }^{40} \mathrm{Ar}+{ }^{58} \mathrm{Ni}(\mathrm{b}=0-3 \mathrm{fm}),{ }^{64} \mathrm{Zn}+{ }^{48} \mathrm{Ti}(\mathrm{b}=2 \mathrm{fm})$, ${ }^{58} \mathrm{Ni}+{ }^{58} \mathrm{Ni}(\mathrm{b}=2.48 \mathrm{fm}),{ }^{64} \mathrm{Zn}+{ }^{58} \mathrm{Ni}(\mathrm{b}=2 \mathrm{fm}),{ }^{86} \mathrm{Kr}+{ }^{93} \mathrm{Nb}(\mathrm{b}=4.07 \mathrm{fm}),{ }^{93} \mathrm{Nb}+{ }^{93} \mathrm{Nb}(\mathrm{b}=3.104$ $\mathrm{fm}),{ }^{129} \mathrm{Xe}+{ }^{\text {Nat }} \mathrm{Sn}(\mathrm{b}=0-3 \mathrm{fm}),{ }^{139} \mathrm{La}+{ }^{139} \mathrm{La}(\mathrm{b}=3.549 \mathrm{fm})$ and ${ }^{197} \mathrm{Au}+{ }^{197} \mathrm{Au}(\mathrm{b}=2.5 \mathrm{fm})$ at their 
corresponding theoretical balance energy ${ }^{1}$ which is, respectively, $119,74,67.3,89.4,67.8,64.6$, $59.3,62.6,56.6,69.2,57,49,51.6$, and $43 \mathrm{MeV} /$ nucleon. The reactions were followed till nuclear transverse flow saturates. As noted from the above, the balance energy is smaller in heavier colliding nuclei, compared to lighter one. As a result, one would expect early saturation in lighter colliding nuclei compared to heavy one. Though, the transverse flow saturates much early in lighter nuclei, some of the quantities, however, keep changing, therefore, we follow all the reactions uniformly till $300 \mathrm{fm} / \mathrm{c}$.

In the following, we shall first study the time evolution and then shall present the mass dependence of different quantities.

\subsection{The Time Evolution}

One of the motivations behind studying a heavy-ion collision is to extract the information regarding the hot and dense nuclear matter. In our approach, the matter density is calculated by 29 ;

$$
\rho(\vec{r}, t)=\sum_{i=1}^{A_{T}+A_{P}} \frac{1}{(2 \pi L)^{3 / 2}} e^{\left(-\left(\vec{r}-\vec{r}_{i}(t)\right)^{2} / 2 L\right)} .
$$

Here $A_{T}$ and $A_{P}$ stand, respectively, for the target and projectile. In actual calculations, we take a sphere of $2 \mathrm{fm}$ radius around the center-of-mass and compute the density at each time step during the reaction using eq. (7). Naturally, one can either extract an average density $\left\langle\rho^{a v g}\right\rangle$ over the whole sphere or a maximal value of the density $\left\langle\rho^{\max }\right\rangle$ reached anywhere in the sphere. In fig. 1(a), we display the $\left\langle\rho^{a v g}\right\rangle / \rho_{0}$ whereas fig. 1(b) shows the $\left\langle\rho^{\max }\right\rangle / \rho_{0}$ as a function of the reaction time. The displayed reactions are ${ }^{20} \mathrm{Ne}+{ }^{27} \mathrm{Al}(\mathrm{A}=47),{ }^{40} \mathrm{Ar}+{ }^{45} \mathrm{Sc}(\mathrm{A}=85),{ }^{64} \mathrm{Zn}+{ }^{58} \mathrm{Ni}$ $(\mathrm{A}=122),{ }^{93} \mathrm{Nb}+{ }^{93} \mathrm{Nb}(\mathrm{A}=186),{ }^{139} \mathrm{La}+{ }^{139} \mathrm{La}(\mathrm{A}=278)$ and ${ }^{197} \mathrm{Au}+{ }^{197} \mathrm{Au}(\mathrm{A}=394)$ spreading

\footnotetext{
${ }^{1}$ The theoretical balance energy was calculated by extrapolating the flow at two different energies with a step of $\pm 10 \mathrm{MeV} /$ nucleon [23].
} 
over the whole mass range. We see that the maximal $\rho^{a v g}$ is slightly higher for lighter systems compared to heavy ones. A similar trend can also be seen for the evolution of $\rho^{\max }$. Further, the maximal value of the density for medium and heavy systems is comparable with the average one. This clearly indicates that the dense matter is formed widely and uniformly in the central region of $2 \mathrm{fm}$ radius. On the other hand, substantial difference in two densities for lighter colliding nuclei indicate that the dense matter is not homogenous and uniform in these reactions. Due to high incident energy, ${ }^{20} \mathrm{Ne}+{ }^{27} \mathrm{Al}$ reaction finishes much early compared to ${ }^{197} \mathrm{Au}+{ }^{197} \mathrm{Au}$ which is simulated at a relatively lower incident energy. Similarly, the peaks in (the maximum $\left\langle\rho^{\max }\right\rangle$ and average $\left.\left\langle\rho^{a v g}\right\rangle\right)$ densities are also delayed in heavier nuclei compared to lighter one. The spreading of the high density zone in heavier colliding nuclei over the long time span indicates the on going interactions among nucleons. This result is in agreement with 29].

Another quantity directly linked with the density is the collision rate. In fig. 2, we display the $d N_{\text {coll }} / d t$ versus time. This rate represents the net collisions after fulfilling the Pauli principle. Naturally, the attempted rate will be much higher than the allowed one. Due to larger interaction volume, the interactions among nucleons in heavy nuclei continue for a long time. This effect should be obvious if one looks the density profile (see fig. 1). A finite density zone will naturally lead to more and more nn collisions and as a result, the collision rate will be more for heavy colliding nuclei.

As stated above, all the reactions are simulated at the balance energy where attractive forces counter balance the repulsive forces. This fact should also be reflected in the quantities like the spectator and participant matter. All nucleons having experienced at least one collision are counted as participant matter. The remaining matter is labeled as the spectator matter. The nucleons with more than one collision are labeled as super-participant matter. This definition gives us possibility to analyze the reaction in terms of participant-spectator fireball model. 
In fig. 3, we display the normalized spectator matter (upper part) and participant matter (lower part) as a function of the time. At the start of the reaction, all nucleons constitute spectator matter. Therefore, no participant matter exists at $t=0 \mathrm{fm} / \mathrm{c}$. Since the ${ }^{20} \mathrm{Ne}+{ }^{27} \mathrm{Al}$ reaction happens at a relative higher energy $(=119 \mathrm{MeV} /$ nucleon $)$, the transition from the spectator to participant matter is swift and fast. On the other hand, due to low bombarding energy, the transition from the spectator to participant matter in heavier colliding nuclei is rather slow and gradual. Interestingly, at the end, all reactions (that happen between incident energy 43 and $119 \mathrm{MeV} /$ nucleon) lead to nearly same participant matter indicating the universality in the balancing of attractive and repulsive forces.

From the above facts, it is clear that heavier colliding nuclei (at $E_{b a l}$ ) have delayed and expanded evolution of the density and participant-spectator matter. It will be of more interest to see how their mass dependence behaves like. This will be discussed in the following paragraphs.

\subsection{The Mass Dependence}

In fig. 4, we display the maximal value of $\left\langle\rho^{a v g}\right\rangle$ and $\left\langle\rho^{\max }\right\rangle$ versus composite mass of the system. Note that all the reactions, considered here, are symmetric i.e. $\eta\left(\left|\frac{A_{T}-A_{P}}{A_{T}+A_{P}}\right|\right)<$ 0.2. Very interesting, we see that the maximal value follows a power law $\propto A^{\tau}$ with $\tau$ being $-0.05182 \pm 0.00776$ for average density $\left\langle\rho^{a v g}\right\rangle$ and $-0.11477 \pm 0.01217$ for maximum density $\left\langle\rho^{\max }\right\rangle .^{2}$ In other words, a slight decrease in the density occurs with the size of the system. This decrease is much smaller compared to the $E_{b a l}\left(\tau_{\text {expt }}=-0.42079 \pm 0.04594\right.$ and $\tau_{\text {th }}=$ $-0.41540 \pm 0.08166)$. Had these reactions being simulated at a fixed incident energy, the trend would have been totally different [29]. Since lighter nuclei cannot be compressed easily, their maximal density at a fixed incident energy will be less compared to heavy nuclei. Since $E_{b a l}$ in

\footnotetext{
${ }^{2} \mathrm{~A}$ small deviation can be seen in the cases where $\eta \neq 0$.
} 
the present case scales as $A^{\tau}$, a weak mass dependence is also observed in density profiles.

The mass dependence of the (allowed) nn collisions is depicted in fig. 5. Here one sees a (nearly) linear enhancement in the nn collisions with the size of the interacting system. This enhancement can be explained mainly using a power law $\propto A^{\tau}$; with $\tau=0.87829 \pm 0.01833$. If one keeps the incident energy fixed (e.g. in the figure, we kept $\mathrm{E}=50 \mathrm{MeV} /$ nucleon), the nn collisions should scale as "A". Our fitting gives $\tau=1.04633 \pm 0.01712$, which is very close to unity.

A dynamical quantity that can serve as an indicator of the role of repulsive and attractive forces is the participant and spectator matter. Naturally, the possibility of a collision will depend upon the mean free path of nucleons. Similar is the case of spectator and participant matter. In fig. 6, we display the spectator, participant and super-participant matter (obtained at $300 \mathrm{fm} / \mathrm{c}$ ) as a function of the total mass of the system. Very interesting, we see a nearly mass independent behavior of the participant matter $(\tau=-0.03621 \pm 0.00954)$. Similar behavior occurs in the case of spectator matter $(\tau=0.08323 \pm 0.02232)$. A slight deviation occurs in the case of ${ }^{20} \mathrm{Ne}+{ }^{27} \mathrm{Al}$. Some small fluctuations can also be due to the variation in the impact parameter, which is not fixed in the present study. The choice of impact parameter is guided by the experimental measurements. As noted in ref. [21, the variation in the impact parameter can have drastic influence on the participant/spectator matter. The super-participant matter shows a little more dependence on the mass of the system $(\tau=-0.14241 \pm 0.04184)$. This can be understood again by looking at the density profile (fig. 4). There one concluded that the lighter nuclei lead to higher densities. In other words, the mean free path will be smaller in lighter nuclei that results in more nn collisions. One should keep in the mind that the mass independent nature of the participant matter is not a trivial observation. For a fixed system mass, the participant matter depends linearly on the incident energy. In the present case, 
though the mass of the system increases, their corresponding energy decreases, resulting in the net mass independent nature. This also suggests that the repulsive and attractive forces at $E_{b a l}$ counter-balance each other in such a manner that the net participant matter remains the same. One may also say that since the contribution of the mean field towards transverse flow is nearly mass independent [23, 30], one needs same amount of participant matter to counter balance the attractive forces. In other words, the participant matter can act as a barometer to study the balance energy in heavy-ion collisions.

The associated quantity linked with the dense matter is the temperature. In principle, a true temperature can be defined only for a thermalized and equilibrated matter. Since in a heavy-ion collision, the matter is non-equilibrated, one can not talk of "temperature". One can, however, look in terms of a local environment only. In our present case, we follow the description of the temperature given in refs. [29, 31, 32. Several different authors have given different descriptions of the local or global temperatures [33, 34, 35, 36]. Some studies of temperature are based on the fireball model [33, whereas others take the hydrodynamical model into account 34]. In refs. 34, 35, the thermalization is directly connected with the non-diagonal elements of the stress tensor. One has even defined the "transverse" temperature in terms of $\left\langle P_{I}^{2} / 2 m\right\rangle ; P_{I}^{2}$ is the average transverse momentum squared [34]. In the present case, the extraction of the temperature " $T$ " is based on the local density approximation i.e. one deduces the temperature in a volume element surrounding the position of each particle at a given time step [29, 31, 32. Here, we postulate that each local volume element of nuclear matter in coordinate space and time has some "temperature" defined by the diffused edge of the deformed Fermi distribution consisting of two colliding Fermi spheres which is typical for a non-equilibrium momentum distribution in heavy-ion collisions.

In this formalism, (dubbed as hot Thomas-Fermi approach [29]), one determines the exten- 
sive quantities like the density, kinetic energy, as well as the entropy with the help of momentum distributions at a given temperature. For more details, reader is referred to refs. [29, 31, 32]. Using this formalism, we also extracted the average and maximum temperature within a central sphere of $2 \mathrm{fm}$ radius as described in the case of density.

In fig. 7, we plot the maximal value of $\left\langle T^{a v g}\right\rangle$ and $\left\langle T^{\max }\right\rangle$ as a function of the composite mass of the system. Some fluctuations can be seen in these plots that can be due to the choice of impact parameter as well as incident energy [29, 32, 34, 37, 38. As stated above, the impact parameter choice is guided by the experimental constraints. Further, the $E_{b a l}$ was extracted using a straight line interpolation, therefore, both these factors may add to the present fluctuations. One sees that both quantities can be parameterized in terms of a power law function $\propto A^{\tau}$; The power factor $\tau$ is rather quite large (being equal to $-0.83743 \pm 0.11355$ and $-0.51079 \pm 0.08218$ ), respectively, for the average and maximal temperature. This sharp mass dependence in the temperature is rather in contradiction to the mild mass dependence obtained in all other quantities. This is not astonishing since temperature depends, crucially, on the kinetic energy (or the excitation energy) of the system [29, 31, 32, 37, 38. It was shown in ref. [37, 38] that for a given colliding geometry, the maximal value of the temperature does not depend upon the size of the interacting source. Rather it depends only on the bombarding energy.

In fig. 8, we display the time of maximal collision rate and average density. We see a power law behavior in both the quantities. The small balance energy in heavy nuclei delays the maximal compression. Interestingly, the power factor $\tau$ is close to $(1 / 3)$ in both the cases. The $E_{b a l}$ was shown to scale with power factor $\tau \approx-0.4$. In other words, the time of maximal collision rate and density varies approximately as a inverse of the $E_{b a l}$.

Apart from the maximal quantities, another interesting quantity is the dense zone at the 
balance energy. This is depicted in fig. 9 where we display the time interval for which $\rho \geq \rho_{0}$ (upper part) and $\rho \geq \rho_{0} / 2$. Again both quantities follow a power law behaviour. Interestingly, the time intervals for the high density have a power law dependence with $\tau=0.33853 \pm 0.05358$ and $\tau=0.46833 \pm 0.04265$, respectively, for $\rho \geq \rho_{0}$ and $\rho \geq \rho_{0} / 2$ which is again very close to the inverse of the mass dependence of $E_{b a l}$. This also points toward the fact that the formation and identification of the fragments is delayed in heavier nuclei compared to lighter nuclei. This conclusion is in agreement with earlier calculations [28, 38].

\section{Summary}

Using the QMD model, we presented the mass dependence of various quantities (such as average and maximum central density, temperature, collision dynamics, participant and spectator matter as well as the time zone for hot and dense nuclear matter) at the energy of vanishing flow $\left(E_{b a l}\right)$. This study was conducted using a hard equation of state along with nn cross-section of $40 \mathrm{mb}$ strength. This combination is reported to explain the experimentally extracted balance energy for large number of cases [23]. Our calculations present several interesting facts:

The reaction saturation time is smaller for lighter nuclei compared to heavy nuclei. The maximal values of density, temperature and collision rate is also shifted accordingly. In all the cases (i.e. in average and maximum central density, temperature, participant and spectator matter etc.), a power law dependence can be seen. The only quantity where power factor $\tau$ is significant (with $\tau \geq|0.2|$ ) is the temperature reached in the central zone. Other quantities are

nearly mass independent. The mass independent nature of the participant matter makes it a good alternate indicator for determining the balance energy. The existence of dense zone scales as inverse of the energy of vanishing flow. 
This work is supported by the grant (No. SP/S2/K-21/96) from Department of Science and Technology, Government of India.

\section{References}

[1] J.J. Molitoris and H. Stöcker, Phys. Lett. B 162, 47 (1985); G.F. Bertsch, W.G. Lynch, and M.B. Tsang, Phys. Lett. B 189, 384 (1987).

[2] C.A. Ogilvie et al., Phys. Rev. C 42, R10 (1990).

[3] D. Krofcheck et al., Phys. Rev. C 46, 1416 (1992).

[4] D.J. Magestro, W. Bauer, O. Bjarki, J.D. Crispin, M.L. Miller, M.B. Tonjes, A.M. Vander Molen, G.D. Westfall, R. Pak, and E. Norbeck, Phys. Rev. C 61, 021602(R) (2000).

[5] D.J. Magestro, W. Bauer, and G.D. Westfall, Phys. Rev. C 62, 041603(R) (2000).

[6] D. Cussol et al., Phys. Rev. C 65, 044604 (2002).

[7] G.D. Westfall et al., Phys. Rev. Lett. 71, 1986 (1993).

[8] J.P. Sullivan et al., Phys. Lett. B 249, 8 (1990).

[9] J.C. Angelique et al., Nucl. Phys. A614, 261 (1997).

[10] D. Krofcheck et al., Phys. Rev. C 43, 350 (1991).

[11] Z.Y. He et al., Nucl. Phys. A598, 248 (1996).

[12] A. Buta et al., Nucl. Phys. A584, 397 (1995). 
[13] R. Pak et al., Phys. Rev. Lett. 78, 1022 (1997); R. Pak, O. Bjarki, S.A. Hannuschke, R.A. Lacey, J. Lauret, W.J. Llope, A. Nadasen, N.T.B. Stone, A.M. Vander Molen, and G.D. Westfall, Phys. Rev. C 54, 2457 (1996); R. Pak et al., ibid. 53, R1469 (1996).

[14] W.M. Zhang et al., Phys. Rev. C 42, R491 (1990); M.D. Partlan et al., Phys. Rev. Lett. 75, 2100 (1995); P. Crochet et al., Nucl. Phys. A624, 755 (1997).

[15] B.A. Li, Phys. Rev. C 48, 2415 (1993).

[16] V. de la Mota, F. Sebille, M. Farine, B. Remaud, and P. Schuck, Phys. Rev. C 46, 677 (1992).

[17] H.M. Xu, Phys. Rev. C 46, R389 (1992); H.M. Xu, Phys. Rev. Lett. 67, 2769 (1991).

[18] H. Zhou, Z. Li, Y. Zhuo, and G. Mao, Nucl. Phys. A580, 627 (1994).

[19] E. Lehmann, A. Faessler, J. Zipprich, R.K. Puri, and S.W. Huang, Z. Phys. A 355, 55 (1996).

[20] S. Soff, S.A. Bass, C. Hartnack, H. Stöcker, and W. Greiner, Phys. Rev. C 51, 3320 (1995).

[21] S. Kumar, M.K. Sharma, R.K. Puri, K.P. Singh, and I.M. Govil, Phys. Rev. C 58, 3494 (1998); S. Kumar, Ph. D. Thesis, Panjab University, Chandigarh (1999).

[22] A.D. Sood and R.K. Puri, Phys. Rev. Lett., (2003)- submitted.

[23] A.D. Sood and R.K. Puri, Phys. Rev. C, (2003)- submitted.

[24] A. Sood and R.K. Puri, Symposium on Nuclear Physics, 45B, 288 (2002); A. Sood and R.K. Puri, VIII Int. Conf. on Nucleus-Nucleus Collisions, Moscow, Russia, June 17-21 (2003)-accepted. 
[25] J. Aichelin, Phys. Rep. 202, 233 (1991).

[26] C. Hartnack, R.K. Puri, J. Aichelin, J. Konopka, S.A. Bass, H. Stöcker, and W. Greiner, Eur. Phys. J A 1, 151 (1998).

[27] G. Peilert, H. Stöcker, W. Greiner, A. Rosenhauer, A. Bohnet, and J. Aichelin, Phys. Rev. C 39, 1402 (1989); S. Kumar, R.K. Puri, and J. Aichelin, Phys. Rev. C 58, 1618 (1998); S. Kumar and R.K. Puri, Phys. Rev. C 58, 2858 (1998).

[28] J. Singh, S. Kumar, and R.K. Puri, Phys. Rev. C 62, 044617 (2000); J. Singh and R.K. Puri, Phys. Rev. C 62, 054602 (2000).

[29] D.T. Khoa, N. Ohtsuka, A. Faessler, M.A. Matin, S.W. Huang, E. Lehmann, and R.K. Puri, Nucl. Phys. A542, 671 (1992).

[30] B. Blättel, V. Koch, A. Lang, K. Weber, W. Cassing, and U. Mosel, Phys. Rev. C 43, $2728(1991)$.

[31] R.K. Puri, N. Ohtsuka, E. Lehmann, A. Faessler, M.A. Matin, D.T. Khoa, G. Batko, and S.W. Huang, Nucl. Phys. A575, 733 (1994).

[32] D.T. Khoa, N. Ohtsuka, M.A. Matin, A. Faessler, S.W. Huang, E. Lehmann, and R.K. Puri, Nucl. Phys. A548, 102 (1992).

[33] G.F. Bertsch and S. Das Gupta, Phys. Rep. 160, 189 (1988).

[34] H. Stöcker and W. Greiner, Phys. Rep. 137, 277 (1986); R. Clare and D. Strottman, Phys. Rep. 141, 177 (1986).

[35] L. Neise, M. Berenguer, C. Hartnack, G. Peilert, H. Stöcker, and W. Greiner, Nucl. Phys. A519, 375c (1990). 
[36] A. Lang, B. Blättel, V. Koch, K. Weber, W. Cassing, and U. Mosel, Phys. Lett. B 245, 147 (1990); A. Lang, B. Blättel, W. Cassing, V. Koch, U. Mosel, and K. Weber, Z. Phys. A 340, 287 (1991).

[37] R.K. Puri, E. Lehmann, A Faessler, and S.W. Huang, J. Phys. G. 20, 1817 (1994).

[38] R.K. Puri, E. Lehmann, N. Ohtsuka, A Faessler, and S.W. Huang, Proceedings of the International Workshop XXII on Gross Properties of Nuclei and Nuclear Excitations, Hirschegg, Austria, 262 (1994). 


\section{Figure Captions:}

Fig. 1: The evolution of (a) average density $\left\langle\rho^{a v g}\right\rangle$ and (b) the maximum density $\left\langle\rho^{\max }\right\rangle$ reached in a central sphere of radius $2 \mathrm{fm}$ as a function of time. Here reactions of ${ }^{20} \mathrm{Ne}+{ }^{27} \mathrm{Al}$ $(b=2.6103 \mathrm{fm}),{ }^{40} \mathrm{Ar}+{ }^{45} \mathrm{Sc}(\mathrm{b}=3.187 \mathrm{fm}),{ }^{64} \mathrm{Zn}+{ }^{58} \mathrm{Ni}(\mathrm{b}=2 \mathrm{fm}),{ }^{93} \mathrm{Nb}+{ }^{93} \mathrm{Nb} \quad(b=3.104 \mathrm{fm})$, ${ }^{139} \mathrm{La}+{ }^{139} \mathrm{La}(\mathrm{b}=3.549 \mathrm{fm})$ and ${ }^{197} \mathrm{Au}+{ }^{197} \mathrm{Au}(\mathrm{b}=2.5 \mathrm{fm})$ are simulated at their corresponding theoretical balance energies (for details, see the text). The shaded area represents the reaction of ${ }^{20} \mathrm{Ne}+{ }^{27} \mathrm{Al}$.

Fig. 2: Same as fig. 1(a), but the rate of allowed collisions $d N_{\text {coll }} / d t$ versus reaction time.

Fig. 3: Same as fig 1, but the time evolution of normalized spectator matter (upper part) and participant matter (lower part).

Fig. 4: The maximal value of the average density $\left\langle\rho^{a v g}\right\rangle_{\max }$ (upper part) and maximum density $\left\langle\rho^{\max }\right\rangle_{\max }$ (lower part) as a function of the composite mass of the system. The solid lines are the fits to the calculated results using $c . A^{\tau}$ obtained with $\chi^{2}$ minimization.

Fig. 5: The total number of the allowed collisions (obtained at the final stage) versus composite mass of the system. The solid squares and open diamonds are the results obtained at $E_{b a l}$ and $50 \mathrm{MeV} /$ nucleon, respectively. The solid and dashed lines are the fits obtained with procedure explained in fig. 4.

Fig. 6: Same as fig. 5, but for the final saturated participant, spectator and super- 
participant matter.

Fig. 7: Same as fig. 5, but for the maximal value of the average temperature (upper part) and maximum temperature (lower part).

Fig. 8: Same as fig. 5, but for the time of maximal value of collision rate (open stars) and average density (solid squares). The solid and dashed lines represent the $\chi^{2}$ fits with power law c. $A^{\tau}$.

Fig. 9: Same as fig. 8, but for the time zone for $\left(\rho \geq \rho_{0}\right)$ (upper part) and for $\left(\rho \geq \rho_{0} / 2\right)$ (lower part) as a function of composite mass of the system. 
Fig 1: "Nuclear Dynamics at the Balance Energy" by Sood and Puri
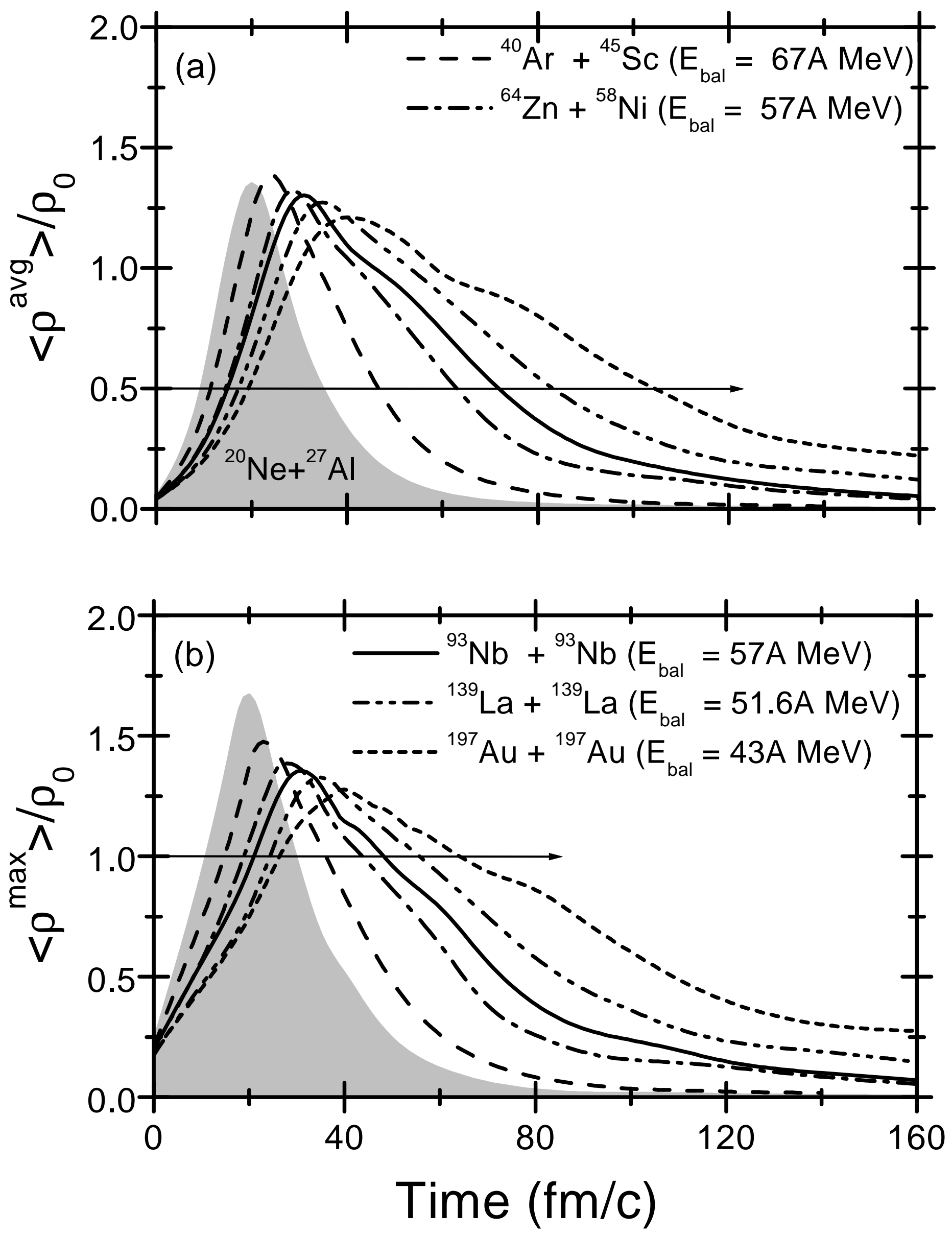


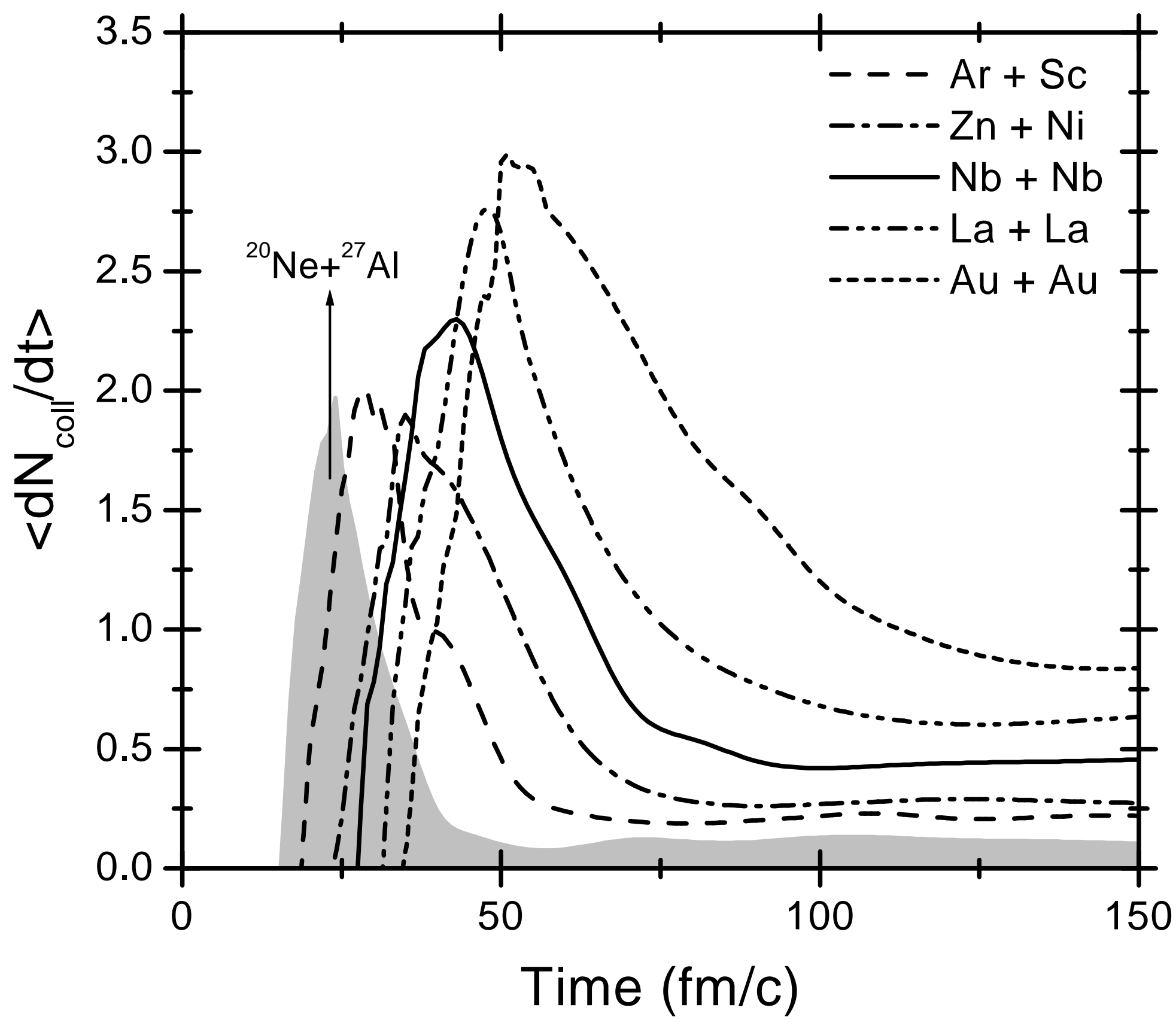

Fig 2: "Nuclear Dynamics at the Balance Energy" by Sood and Puri 
Fig 3: "Nuclear Dynamics at the Balance Energy" by Sood and Puri
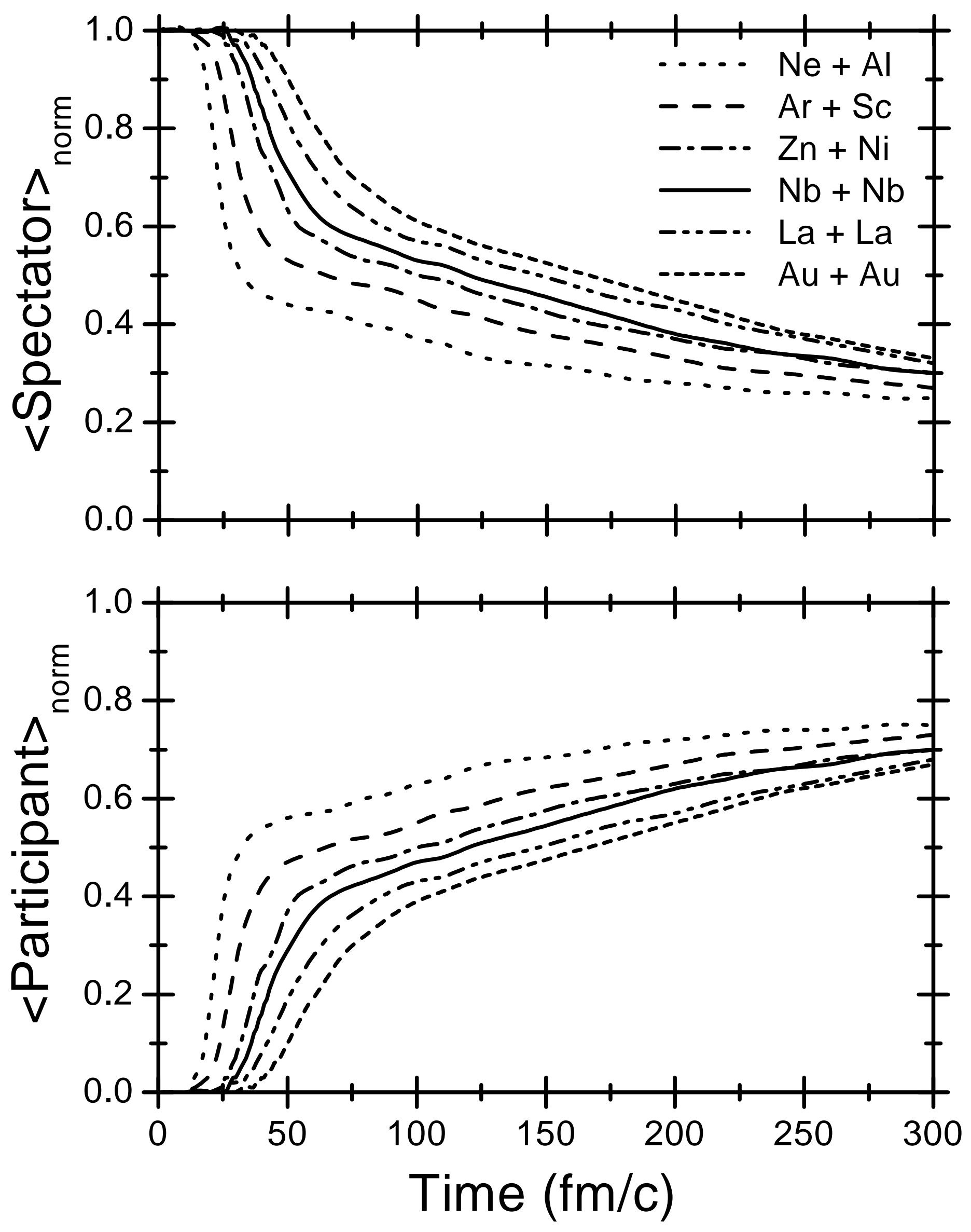
Fig 4: "Nuclear Dynamics at the Balance Energy" by Sood and Puri
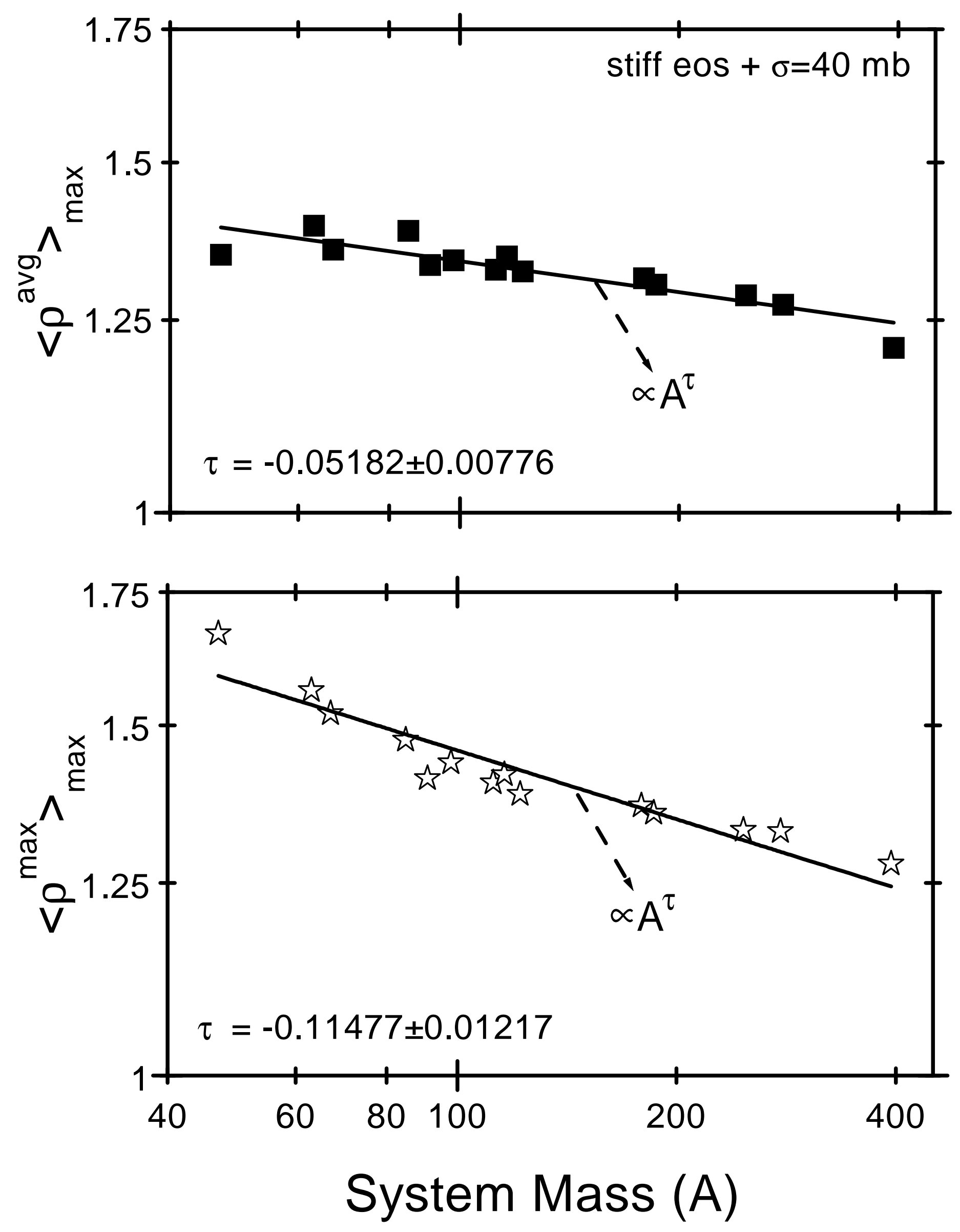


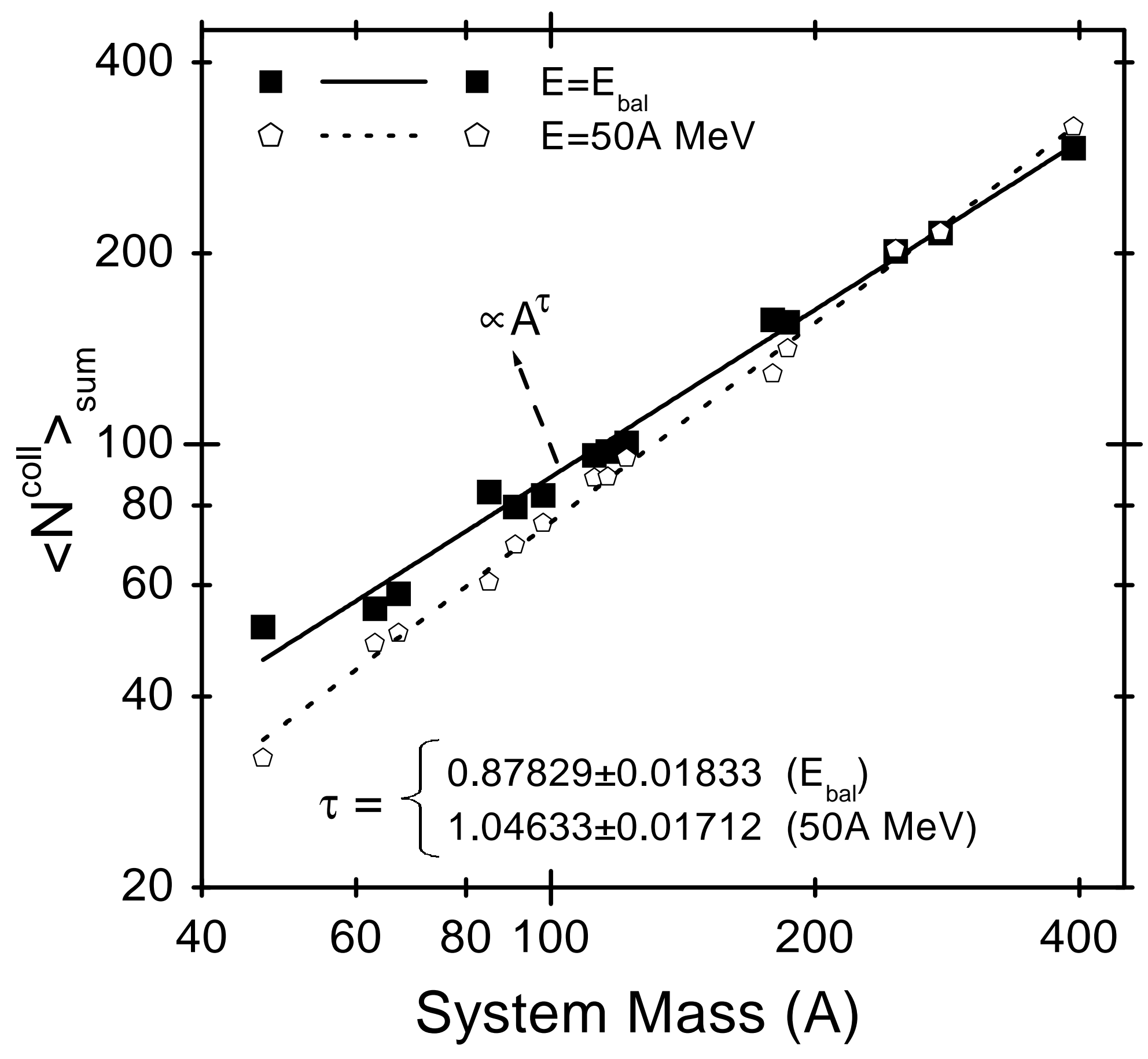

Fig 5: "Nuclear Dynamics at the Balance Energy" by Sood and Puri 


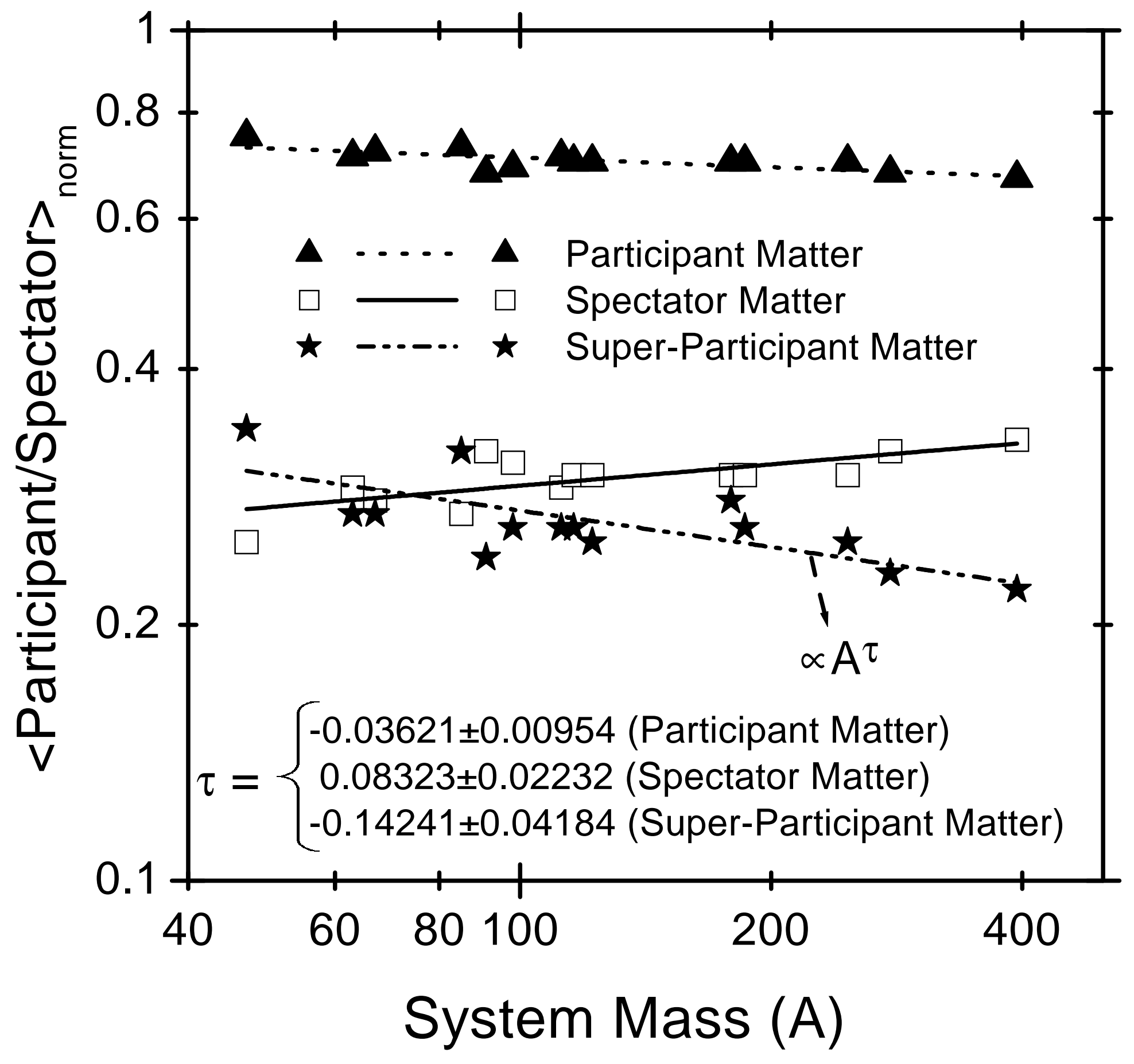

Fig 6: "Nuclear Dynamics at the Balance Energy" by Sood and Puri 
Fig 7: "Nuclear Dynamics at the Balance Energy" by Sood and Puri
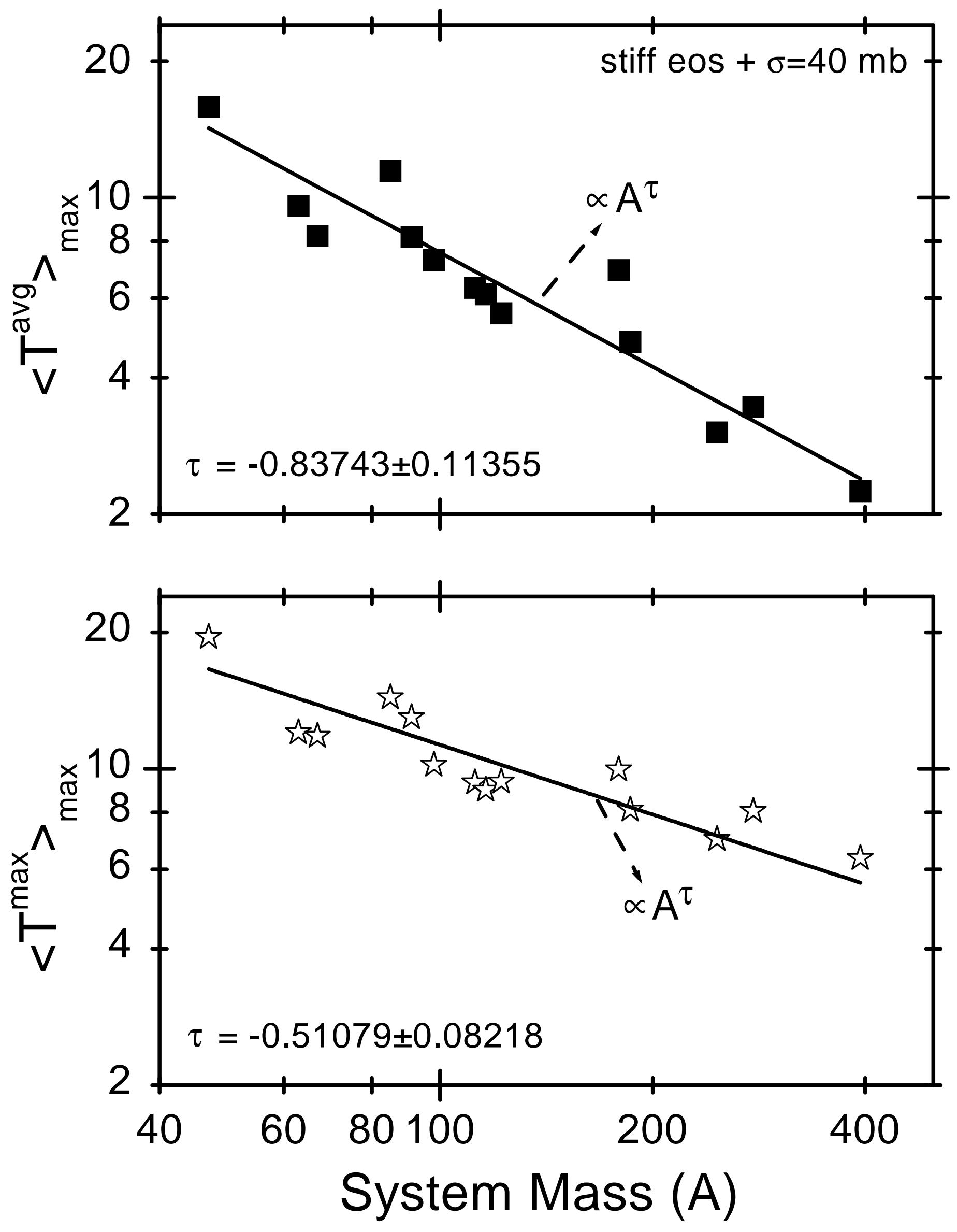


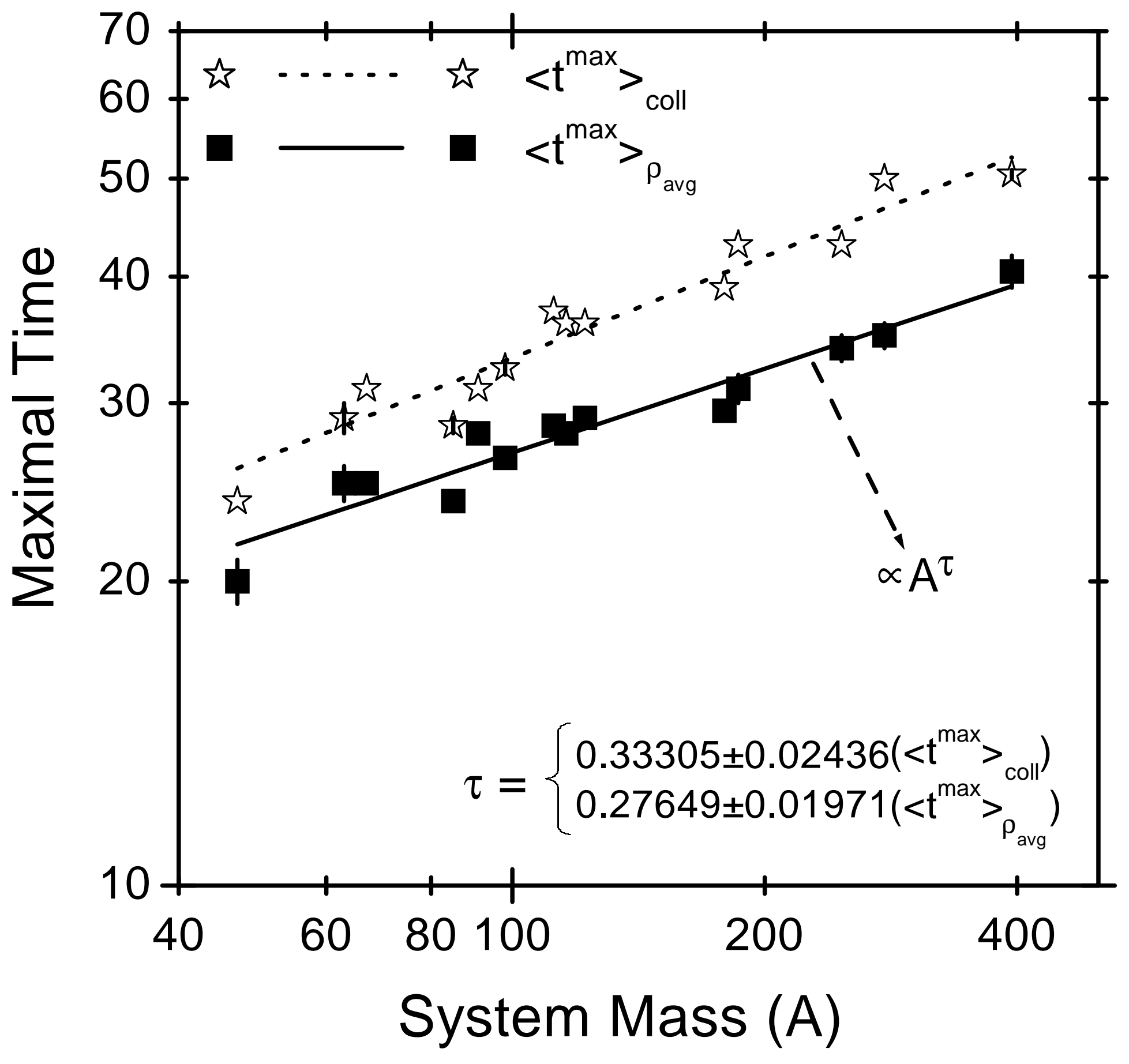

Fig 8: "Nuclear Dynamics at the Balance Energy" by Sood and Puri 
Fig 9: "Nuclear Dynamics at the Balance Energy" by Sood and Puri
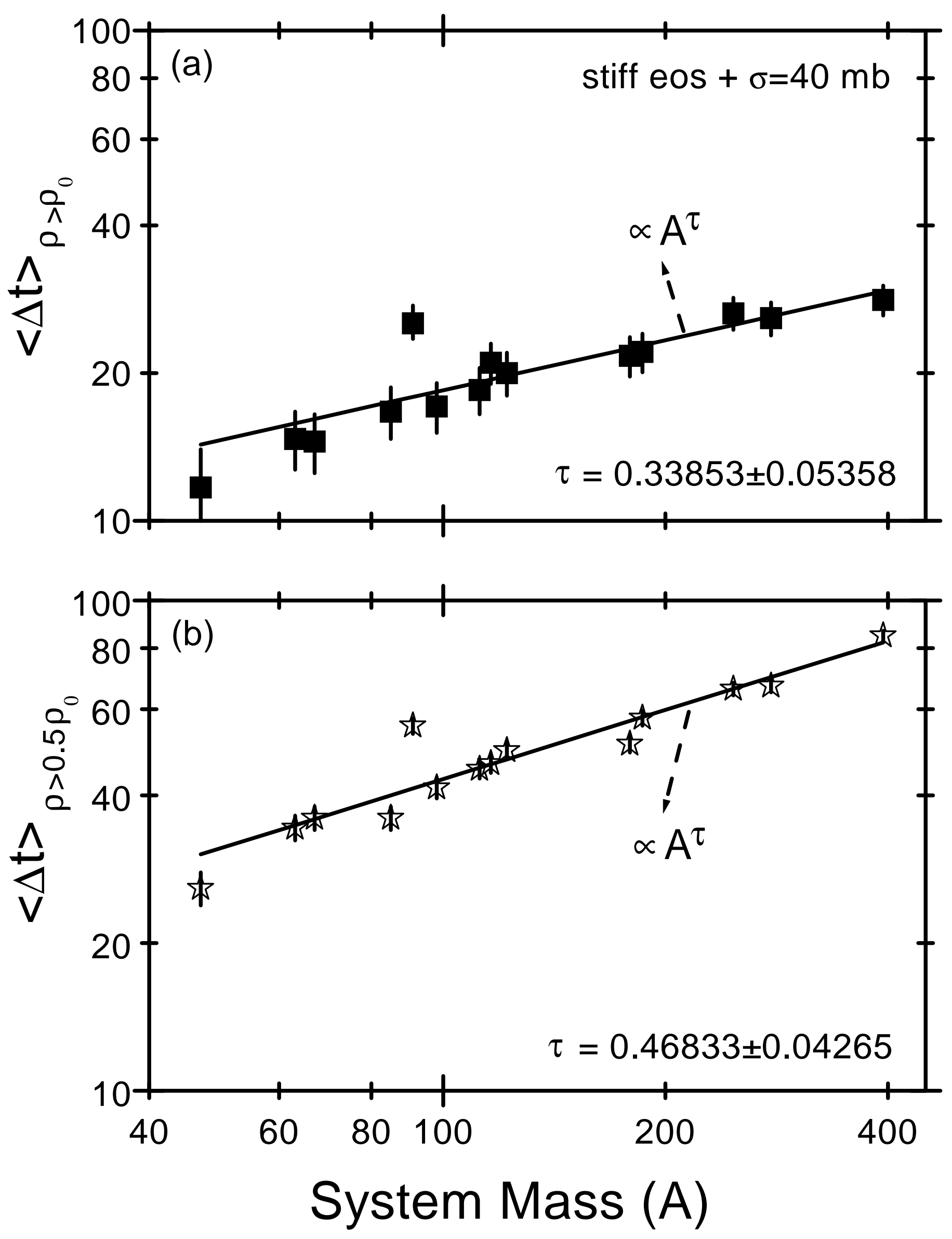